\title{
Non-monochromatic whistler waves detected by Kaguya on the dayside surface of the moon
}

\author{
Tomoko Nakagawa ${ }^{1}$, Futoshi Takahashi ${ }^{2}$, Hideo Tsunakawa ${ }^{2}$, Hidetoshi Shibuya $^{3}$, \\ Hisayoshi Shimizu ${ }^{4}$, and Masaki Matsushima ${ }^{2}$ \\ ${ }^{1}$ Tohoku Institute of Technology, 35-1 Yagiyama Kasumi-cho, Taihaku-ku, Sendai, Miyagi 982-8577, Japan \\ ${ }^{2}$ Department of Earth and Planetary Sciences, Tokyo Institute of Technology, 2-12-1 Ookayama, Meguro-ku, Tokyo 152-8550, Japan \\ ${ }^{3}$ Department of Earth Sciences, Kumamoto University, 39-1, Kurokami 2-chome, Kumamoto 860-8555, Japan \\ ${ }^{4}$ Earthquake Research Institute, University of Tokyo, 1-1-1, Yayoi, Bunkyo-ku, Tokyo 113-0032, Japan
}

(Received August 9, 2009; Revised December 28, 2009; Accepted January 19, 2010; Online published February 21, 2011)

\begin{abstract}
Non-monochromatic uctuations of the magnetic eld over the frequency range of $0.03-10 \mathrm{~Hz}$ were detected by Kaguya at an altitude of $100 \mathrm{~km}$ above the lunar surface. The uctuations were almost always observed on the solar side of the moon, irrespective of the local lunar crustal eld. They were also detected just nightside of the terminator $\left(\mathrm{SZA}<123^{\circ}\right)$, but were absent around the center of the wake. The level of the uctuation enhanced over the wide range from 0.03 to $10 \mathrm{~Hz}$, with no clear peak frequency. The uctuations had the compressional component, and the polarization was not clear. The uctuations were supposed to be whistler waves generated by the protons re ected by the lunar surface. The re ected protons are scattered in various directions, resulting a wide range of distribution of the velocity component parallel to the magnetic eld. It may account for the wide range of frequency as observed, through cyclotron resonance of the wave with the re ected ions, in which the resonant frequency depends on the velocity component parallel to the magnetic eld. However, there is also the possibility that the waves were generated by some nonresonant process.
\end{abstract}

Key words: Moon, whistler wave, solar wind, re ected protons, Kaguya.

\section{Introduction}

Since the moon does not have a global magnetic eld system, the solar wind particles can access the lunar surface directly. This results in the solar wind interaction with the moon being quite different from that with the Earth.

Absorption of the solar wind particles by the lunar surface leads to the formation of the lunar wake, a plasma cavity in the solar wind left on the anti-solar side of the moon (Colburn et al., 1967; Lyon et al., 1967; Ness et al., 1968; Schubert and Lichtenstein, 1974; Ogilvie et al., 1996; Owen et al., 1996; Bosqued et al., 1996). It has recently been determined that not all the solar wind particles are absorbed, but that $0.1-1 \%$ of the solar wind protons are reected by the lunar surface (Saito et al., 2008). Some of the re ected protons can also access the center of the near wake due to their large Larmour radius (Nishino et al., 2009).

The solar wind interaction with the moon generates wave activities around the moon. WIND and Geotail spacecraft detected monochromatic whistler waves in the solar wind when the spacecraft were magnetically connected with the lunar wake (Farrell et al., 1996; Nakagawa et al., 2003). Monochromatic whistler waves were also detected by Lunar Prospector in association with the lunar external magnetic enhancements (LEME) or the lunar crustal magnetic eld

Copyright (C) The Society of Geomagnetism and Earth, Planetary and Space Sciences (SGEPSS); The Seismological Society of Japan; The Volcanological Society of Japan; The Geodetic Society of Japan; The Japanese Society for Planetary Sciences; TERRAPUB.

doi:10.5047/eps.2010.01.005
(Halekas et al., 2006a). These were circularly polarized waves propagating along the magnetic eld against the solar wind ow. Their generation has been related to eldaligned beams of ions or electrons.

Such monochromatic whistler waves are signi cant in waveforms or in spectra, but they are rarely observed. Instead, most commonly observed around the moon are (1) large-amplitude, low-frequency waves with a period of around $100 \mathrm{~s}$, and (2) non-monochromatic uctuations whose frequencies range from 0.03 to $10 \mathrm{~Hz}$. Figure 1 shows an example of the dynamic spectrum for a 24-h period on January 4, 2008, obtained by the Kaguya/LMAG magnetometer on its orbit around the moon. As well as intense low-frequency waves at around $0.01 \mathrm{~Hz}$, broadband magnetic uctuations are observed repeatedly in the range from $0.03 \mathrm{~Hz}$ up to about $5 \mathrm{~Hz}$. Similar sporadic, broadband $(0-2 \mathrm{~Hz})$ magnetic turbulence was observed by Lunar Prospector when the spacecraft was magnetically connected to the surface of the moon (Halekas et al., 2006b).

The aim of this paper is to present the properties of the non-monochromatic waves observed mainly on the dayside surface of the moon, and to discuss their generation mechanism.

\section{Observation}

The magnetic eld data used in this study were obtained by MAP-LMAG magnetometer (Shimizu et al., 2008; Takahashi et al., 2009) onboard Kaguya on its orbit encircling the moon at an altitude of $100 \mathrm{~km}$. Figure 2 shows an example of the orbit of Kaguya on March 8, 2008, 


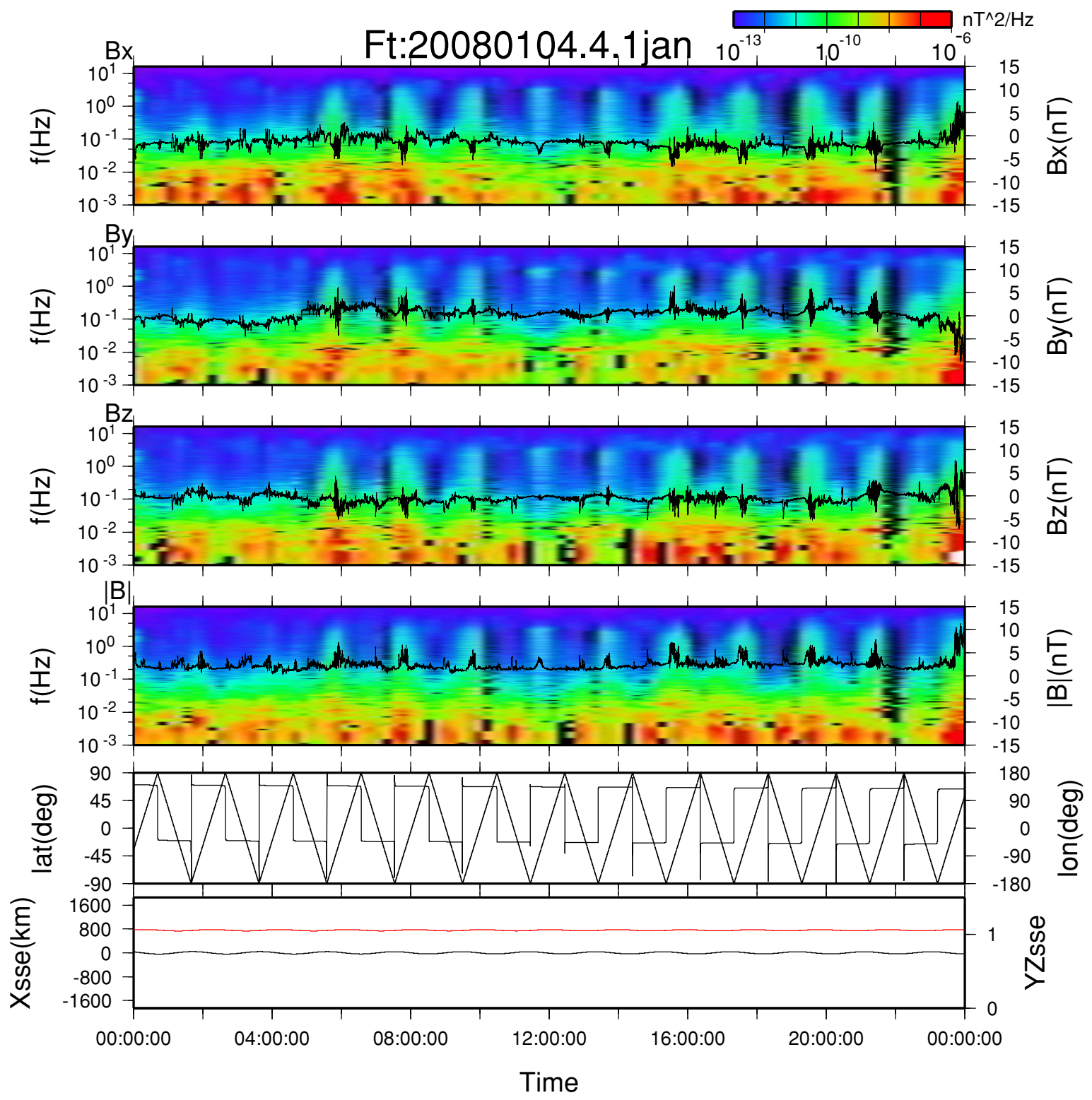

Fig. 1. The dynamic spectra of the magnetic field components $B_{x}, B_{y}, B_{z}$ in SSE coordinates, and the magnitude $|\mathbf{B}|$ obtained by KAGUYA MAP-PACE on January 4, 2008. Overlaid are the magnetic field components and the magnitude. The magnetic field data sampled at $32 \mathrm{~Hz}$ were Fourier transformed every $1024 \mathrm{~s}$. The bottom two panels show the latitude and the longitude of Kaguya in the mean Earth/polar axis (ME) coordinate system, and the $x$-component of the position in selenocentric solar ecliptic (SSE) coordinates together with the distance $Y Z_{\text {sse }}=\sqrt{Y_{\text {sse }}^{2}}+Z_{\text {sse }}^{2}$ normalized by the lunar radius (red curve; $Y Z_{\text {sse }}>1$ indicates that the spacecraft was not shadowed by the moon). The orbit of Kaguya was nearly along the terminator on this day.

when the moon was in the solar wind. The period of the orbital motion was about $118 \mathrm{~min}$ at this altitude. The magnetic field vectors were obtained with sampling frequency of $32 \mathrm{~Hz}$.

Figure 3 shows an example of the magnetic fluctuations observed on the orbit shown in Fig. 2. The curves on the top four panels of Fig. 3 appear to be broad during the period from $4: 10$ to $5: 30$ due to the magnetic fluctuations. The spacecraft was on the solar side of the moon during the period from 4:20 to 5:20. The magnetic fluctuations started at $4: 10$, before the spacecraft got out of the lunar wake, and persisted until 5:30, when the spacecraft had entered into the lunar wake, as recognized in Fig. 2(c). That is, the fluctuations were observable in the area where the solar zenith angle was less than about $123^{\circ}$ and were not observed deep in the wake.

The bottom panel of Fig. 3 is an example of the dynamic spectrum of the $B_{y}$ component. The level of fluctuations enhanced during the period from $4: 10$ to $5: 30$. The frequency extended up to $5 \mathrm{~Hz}$ for the period 4:10-5:10, and then up to $10 \mathrm{~Hz}$ for $5: 10-5: 27$. Although the fluctuations were most intense in the weak-field region around 5:20-5:27, just behind a LEME observed at 5:10-5:20, for most of the time $(4: 10-5: 10)$, the magnetic fluctuations were observed far from LEME and seem to have nothing to do with them. Red (blue) bars at the bottom of the spectrum indicate that the spacecraft was magnetically connected with the dayside (nightside) surface of the moon. It should be noted that the spacecraft was not necessarily connected with the lunar surface when the magnetic fluctuations were observed, sug- 

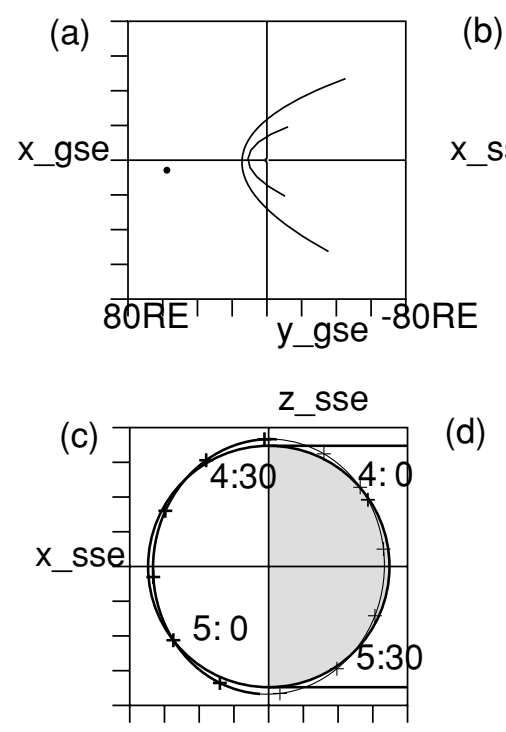

(b)

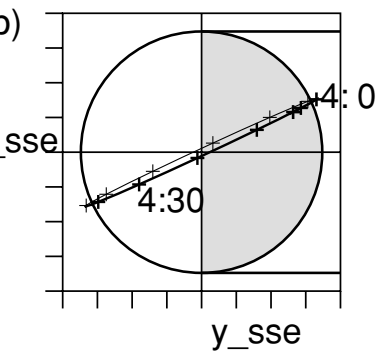

(d)

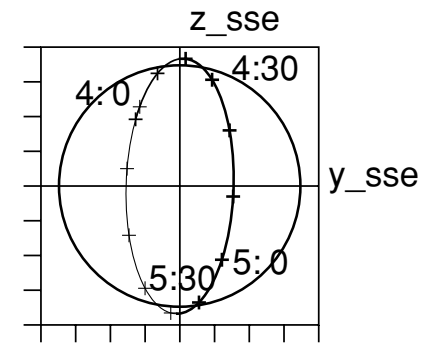

Fig. 2. An example of the orbit of Kaguya on March 8, 2008. (a) The position of the moon together with Kaguya, with respect to the Earth and the nominal bow shock. (b-d) The trajectory of Kaguya encircling the moon, projection onto the (b) $x-y$, (c) $x-z$, and (d) $y-z$ plane of SSE coordinates. Thin-lined curves show that the spacecraft was behind the moon.
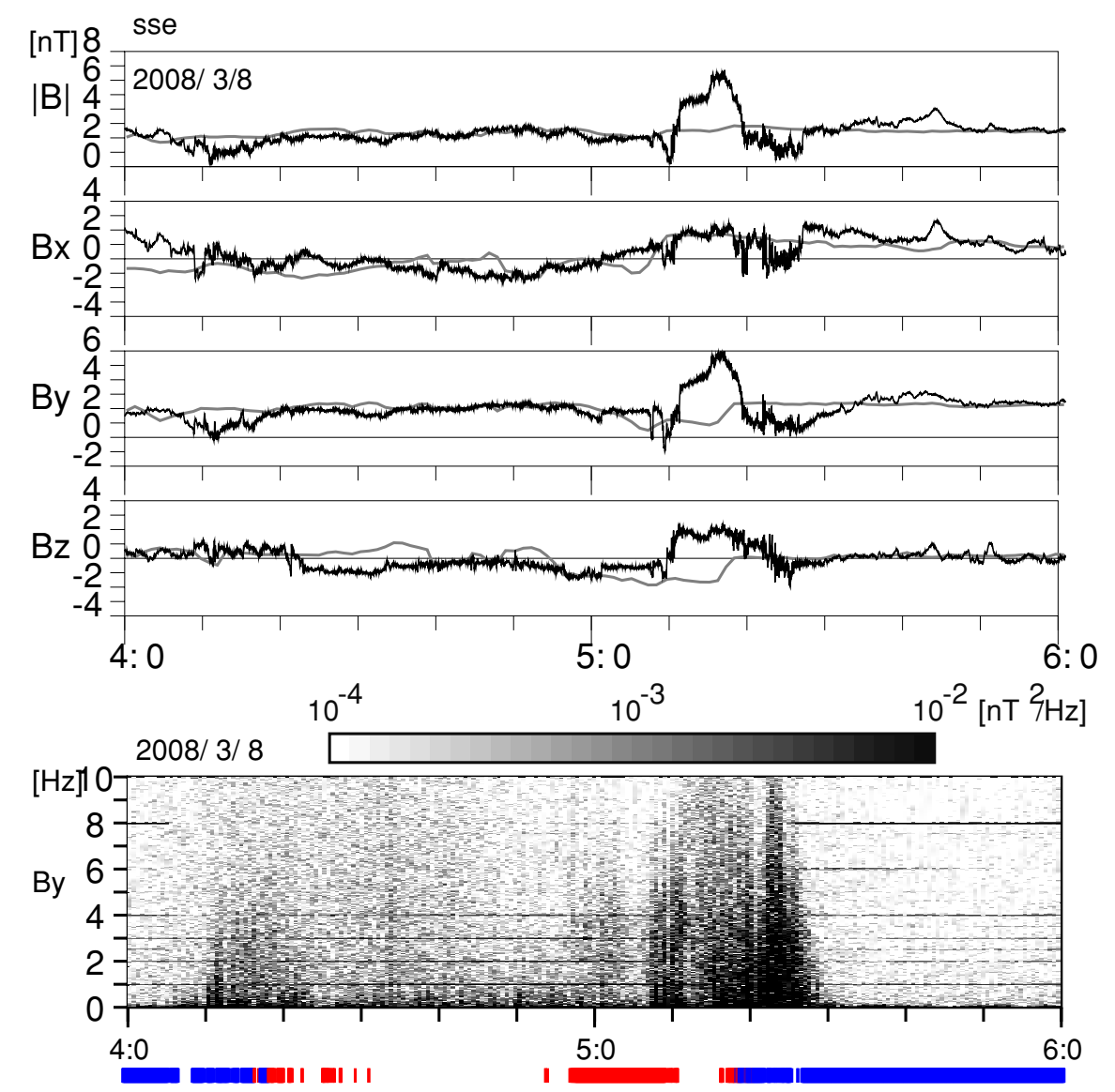

Fig. 3. An example of the magnetic fluctuations observed on March 8, 2008 on the orbit shown in Fig. 2. The four top panels are 1-s averages of the magnetic field components $B_{x}, B_{y}$, and $B_{z}$ in the SSE coordinate system. Gray curves are the solar wind magnetic field observed by ACE, shifted to the Kaguya position by using the solar wind speed. ACE data are in GSE coordinates, but the difference between SSE and GSE is negligible. Bottom panel is the dynamic spectrum of the $B_{y}$ component obtained at a 32-Hz sampling period and Fourier transformed every $32 \mathrm{~s}$.

gesting that the wave propagation was not exactly parallel to the magnetic field.

Figure 4 shows the spectra of the magnetic fluctuations for five different periods selected from Fig. 3. Vertical lines indicate ion cyclotron frequencies $(\sim 0.03 \mathrm{~Hz}$ for periods (a), (b), (e); $0.06 \mathrm{~Hz}$ for (c); $0.02 \mathrm{~Hz}$ for (d)). Compared with the quiet period Fig. 4(e) when Kaguya was in the center of the wake, the level of the fluctuation was enhanced 
(a) $4: 15-4: 20$

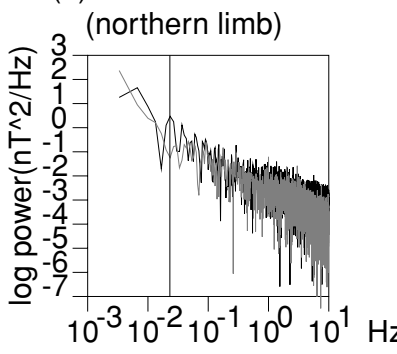

(c) $5: 15-5: 20$

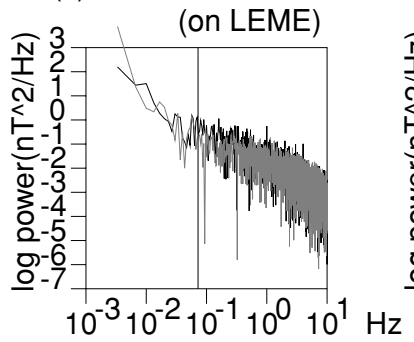

(e) $5: 45-5: 50$

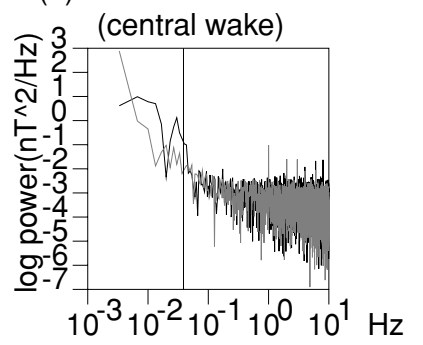

(b) $4: 45-4: 50$

(dayside)

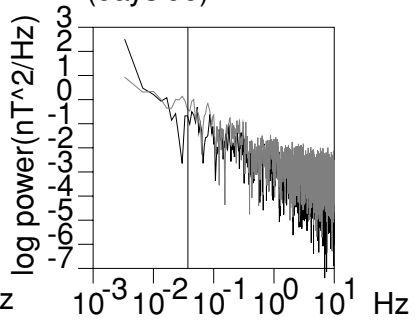

(d) $5: 22-5: 27$

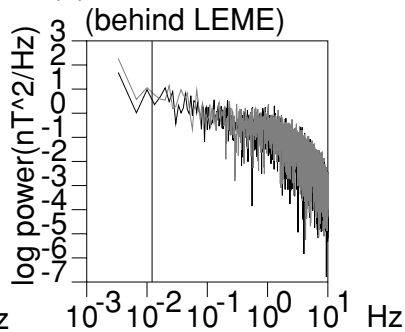

$10^{-3} 10^{-2} 10^{-1} 10^{0} 10^{1} \mathrm{~Hz}$ 


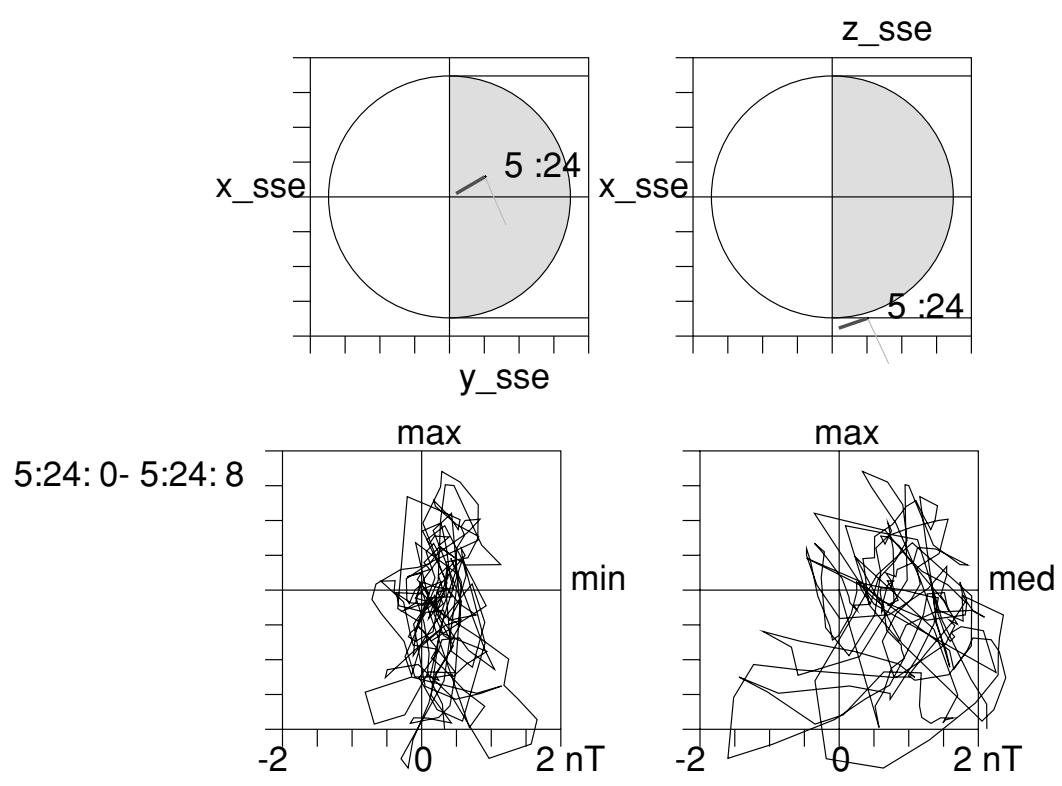

Fig. 5. An example of the minimum variance method applied to the period 5:24:0-5:24:8. Top: the direction of the minimum variance (thick bars) and the average magnetic field (thin lines) projected onto the $x-y$ and $x-z$ plane of the SSE coordinates. Bottom: The hodograms of the magnetic field. The minimum, medium, and maximum variances were $0.15,0.59$ and 0.73 , respectively.

on the solar side of the moon and also detected just nightside of the terminator $\left(\mathrm{SZA}<123^{\circ}\right)$, but they were absent around the center of the wake.

iii) There was no clear peak frequency.

iv) The fluctuations had the compressional component. They were not propagating along the background magnetic field lines.

v) They showed no preferred polarity.

\section{Discussion}

\subsection{Mode of the wave}

The observed frequency $0.03-10 \mathrm{~Hz}$ is the result of the Doppler shift

$$
\omega_{\mathrm{obs}}=\omega+\mathbf{k} \cdot \mathbf{V}_{\mathrm{sw}}
$$

as the spacecraft was in the solar wind flow and observed the waves propagating in the solar wind plasma. If we assume the waves are generated at the lunar surface and propagate upstream against the solar wind, the observed frequency $\omega_{\text {obs }}$ must have been downshifted as $\omega_{\text {obs }}=$ $\omega-\left|\mathbf{k} \cdot \mathbf{V}_{\text {sw }}\right|$, with the wave number vector $\mathbf{k}$ and the solar wind velocity $\mathbf{V}_{\mathrm{sw}}$ in opposite directions. For the observed frequency $(0.03-10 \mathrm{~Hz})$ to be higher than the ion cyclotron frequency $(0.03 \mathrm{~Hz})$, the frequency must have been much higher than the cyclotron frequency in the solar wind frame of reference. The only wave that can present in the frequency range is the whistler wave. In addition, only the whistler mode can propagate against the solar wind with a group velocity larger than the solar wind speed.

If we assume the waves are generated at upstream of Kaguya, the observed frequency would depend on the direction of propagation. Waves propagating upstream would be convected down if the group velocity is smaller than the solar wind flow. They would then be detected at lower frequencies. On the other hand, if the waves were propagating down the solar wind flow, they would be observed at higher frequencies due to the Doppler shift. One might expect that some low-frequency waves, such as magnetohydrodymanic (MHD) waves, might have been detected by Kaguya at a higher frequency, however, these can not account for the frequency range up to $10 \mathrm{~Hz}$ as observed. Substituting $k=\omega / V_{\text {ph }}$ into Eq. (1), where the phase speed $V_{\text {ph }}$ of a MHD wave is of the order of Alfvén speed or sound speed, $V_{\mathrm{ph}} \sim 0.1 V_{\mathrm{sw}}$, we obtain $\omega_{\mathrm{obs}}$ of the order of $10 \omega$. As the frequency of a MHD wave is smaller than the ion cyclotron frequency $\Omega_{i} \sim 0.03 \mathrm{~Hz}$ for this period, the frequency $\omega_{\text {obs }}$ to be detected by Kaguya cannot exceed $0.3 \mathrm{~Hz}$. Therefore, we conclude that a MHD wave is not an appropriate candidate for the non-monochromatic waves of $0.03-10 \mathrm{~Hz}$ as detected by Kaguya. Rather, it is the low-frequency waves at around $0.01 \mathrm{~Hz}$ that are explained by the Doppler shift of the MHD waves.

Given the observed frequency range up to $10 \mathrm{~Hz}$, the wave detected by Kaguya is supposed to be a whistler mode wave. One may think it inconsistent that the nonmonochromatic waves were not propagating parallel to the magnetic field while the monochromatic whistlers reported in earlier publications were propagating parallel to the magnetic field (Farrell et al., 1996; Nakagawa et al., 2003). However, Halekas et al. (2006b) reported that the monochromatic whistler waves in the close vicinity of the moon propagated at all angles to the magnetic field with the largest percentage near $\sim 40^{\circ}$. Furthermore, the propagation direction of a whistler wave tends to be more and more parallel to the magnetic field as it propagates, because the group velocity is more parallel to the magnetic field than the phase velocity. It might then be detected as propagating parallel to the magnetic field at a long distance from the generation site.

\subsection{Possible generation mechanism}

It would be natural to think that the waves were associated with the plasma particles reflected by the lunar surface, 
Table 1. Cycrotron resonance with reflected particles.

\begin{tabular}{|c|c|c|c|c|}
\hline Generation site & $\mathbf{k}$ & Detection by s/c & Resonance condition (fundamental) & Remark \\
\hline \multirow[t]{3}{*}{$\begin{array}{l}\text { Downstream of } \\
\text { s/c }\end{array}$} & Sunward & $\begin{array}{l}\text { Accessible if } \\
V_{\mathrm{g}} \cos \theta_{k S}>V_{\mathrm{sw}}\end{array}$ & $\omega-\left|k\left(V_{e \|}-V_{\mathrm{sw} \|}\right) \cos \theta_{k B}\right|=\Omega_{e}$ & $\begin{array}{l}\text { Impossible as } \omega< \\
\Omega_{e}\end{array}$ \\
\hline & & & $\omega-\left|k\left(V_{i \|}-V_{\mathrm{sw} \|}\right) \cos \theta_{k B}\right|=-\Omega_{i}$ & OK \\
\hline & $\begin{array}{l}\text { Anti- } \\
\text { sunward }\end{array}$ & Inaccessible to $\mathrm{s} / \mathrm{c}$ & & $\begin{array}{l}\text { (Accessible only on } \\
\text { the limb) }\end{array}$ \\
\hline \multirow[t]{4}{*}{ Upstream of s/c } & Sunward & $\begin{array}{l}\text { Accessible if } \\
V_{\mathrm{g}} \cos \theta_{k S}<V_{\mathrm{sw}} \\
\text { (convected down) }\end{array}$ & $\omega-\left|k\left(V_{e \|}-V_{\mathrm{sw} \|}\right) \cos \theta_{k B}\right|=\Omega_{e}$ & $\begin{array}{l}\text { Impossible as } \omega< \\
\Omega_{e}\end{array}$ \\
\hline & & & $\omega-\left|k\left(V_{i \|}-V_{\mathrm{sw} \|}\right) \cos \theta_{k B}\right|=-\Omega_{i}$ & OK \\
\hline & $\begin{array}{l}\text { Anti- } \\
\text { sunward }\end{array}$ & Accessible & $\omega+\left|k\left(V_{e \|}-V_{\mathrm{sw} \|}\right) \cos \theta_{k B}\right|=\Omega_{e}$ & $\begin{array}{l}\text { Not likely as } \\
\omega_{\text {obs }} \ll \Omega_{e}\end{array}$ \\
\hline & & & $\omega+\left|k\left(V_{i \|}-V_{\mathrm{sw} \|}\right) \cos \theta_{k B}\right|=-\Omega_{i}$ & $\begin{array}{l}\text { Impossible } \\
\omega_{\text {obs }}>0\end{array}$ \\
\hline
\end{tabular}

$\theta_{k S}:$ the angle between the wave number vector $\mathbf{k}$ and the solar wind velocity $\mathbf{V}_{\mathrm{sw}}$.

as the waves were mainly observed on the solar side surface of the moon.

A reflected particle with the velocity $\mathbf{V}_{p}$ measured in the rest frame would be injected into the solar wind with a velocity $\left(\mathbf{V}_{p}-\mathbf{V}_{\mathrm{sw}}\right)$ in the solar wind frame of reference (Fig. 6). If we assume the particles to be ions or electrons reflected by or emitted by the surface of the moon, they would start gyrating after the injection into the solar wind magnetic field, and the velocity component parallel to the magnetic field $\left(V_{p \|}-V_{\mathrm{sw} \|}\right) \equiv\left(\mathbf{V}_{p}-\mathbf{V}_{\mathrm{sw}}\right) \cdot \hat{\mathbf{e}}$ would contribute to the Doppler shift of the whistler waves as observed from the particles, where $\hat{\mathbf{e}} \equiv \mathbf{B}_{0} /\left|\mathbf{B}_{0}\right|$ is the unit vector parallel to the background magnetic field.

In the following sections, we discuss the generation mechanism of the whistler wave.

\subsection{Cyclotron resonance}

First, we examine a cyclotron resonance of the solar wind particles reflected by the lunar surface. Table 1 lists the possible resonance conditions of the whistler waves and reflected particles.

The condition of the cyclotron resonance of the upstream-propagating whistler wave and the reflected particles would be

$$
\omega-\left|k\left(V_{e \|}-V_{\mathrm{sw} \|}\right) \cos \theta_{k B}\right|=\Omega_{e}
$$

for electrons $(p=e)$, and

$$
\omega-\left|k\left(V_{i \|}-V_{\mathrm{sw} \|}\right) \cos \theta_{k B}\right|=-\Omega_{i}
$$

for ions $(p=i)$, where $\Omega_{i}$ is the ion cyclotron frequency and $\theta_{k B}$ is the angle between the wave number vector $\mathbf{k}$ and the magnetic field $\mathbf{B}_{0}$. Note that the signs of $V_{e \|}$ and $V_{i \|}$ are negative when $V_{\mathrm{sw} \|}$ is positive, as the particles are reflected. The negative sign on the ion cyclotron frequency $\Omega_{i}$ indicates that the rotation (left-handed) is in opposite direction

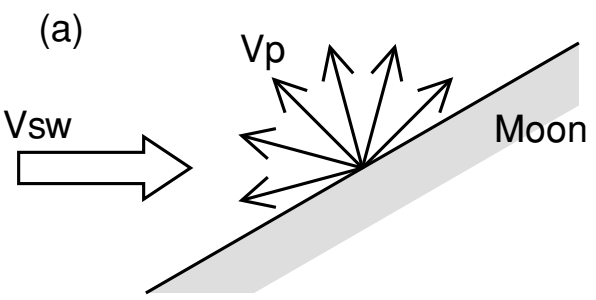

(b)

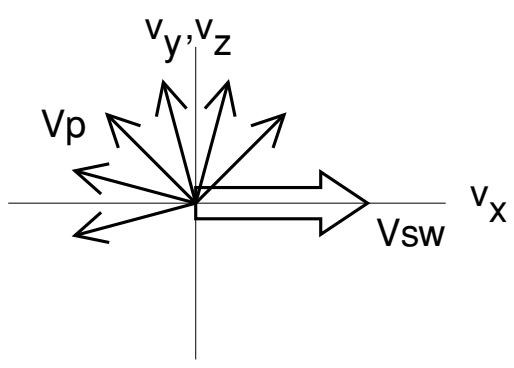

(c)

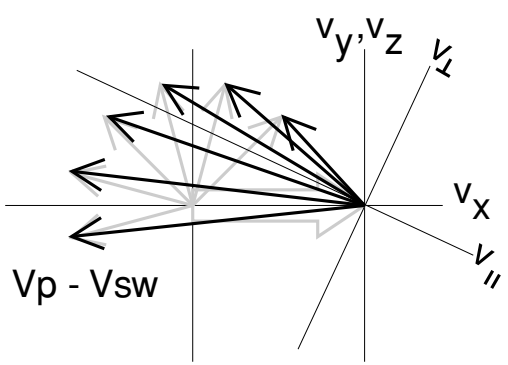

Fig. 6. Schematic illustrations of (a) the velocity of an incident solar wind and the reflected particles scattered into various directions, (b) the velocity distribution of the solar wind and the reflected particles in the rest frame fixed on the lunar surface, (c) the velocity distribution of the reflected particles in the solar wind frame of reference. 
from the rotation of the whistler wave (right-handed) in the solar wind frame. The resonance with electrons (Eq. (2)) is impossible because the angular frequency $\omega$ of the whistler wave is smaller than the electron cyclotron frequency $\Omega_{e}$. Subtracted by $\left|k\left(V_{e \|}-V_{\mathrm{sw} \|}\right) \cos \theta_{k B}\right|$, the angular frequency can never become equal to $\Omega_{e}$.

The resonance condition for downstream-propagating waves are

$$
\omega+\left|k\left(V_{e \|}-V_{\mathrm{sw} \|}\right) \cos \theta_{k B}\right|=\Omega_{e}
$$

for electrons $(p=e)$, and

$$
\omega+\left|k\left(V_{i \|}-V_{\mathrm{sw} \|}\right) \cos \theta_{k B}\right|=-\Omega_{i}
$$

for ions $(p=i)$. Again, the resonance condition (4) for electrons is not likely because the observed frequency $\omega_{\mathrm{obs}}=\omega+\left|k V_{\mathrm{sw} \|} \cos \theta_{k B}\right|$ is much smaller than $\Omega_{e}$ and the magnitude of $\left|k V_{e \|} \cos \theta_{k B}\right|$, which is equal to or smaller than $\left|k V_{\mathrm{sw} \|} \cos \theta_{k B}\right|$, is also too small to match $\Omega_{e}$.

Accordingly, we conclude that the resonant particles must be protons if we assume cyclotron resonance as a generation mechanism of the whistler wave. A famous example of whistler waves generated through the cyclotron resonance with ions is the upstream waves at the Earth's bow shock (Fair eld, 1974). There are two major frequency ranges of the waves upstream from the Earth's bow shock, $0.01-0.05 \mathrm{~Hz}$ and $0.5-4 \mathrm{~Hz}$. The former is thought to be MHD waves generated by the ions that are re ected by the bow shock to reach far upstream of the observer because of the large Larmour radius and then convected down by the solar wind ow (see Fair eld, 1969; Russell, 1994a, b). The latter is the whistler waves generated at the bow shock by the re ected ions, which propagate against the solar wind. The two frequency ranges seem to be analogous to those observed near the moon. The Earth's bow shock is a huge obstacle as seen from the perspective of the solar wind ions. We need to examine whether the moon re ects a signi cant number of solar wind ions.

Figure 7 compares the non-monochromatic waves with the re ected protons detected by the MAP-PACE Ion Mass Analyzer (IMA) onboard Kaguya (Saito et al., 2008). It has been reported that $0.1-1 \%$ of the solar wind protons are reected back from the moon instead of being absorbed by the lunar surface. Enhancement of magnetic uctuations occurred during the period from $12: 27$ to $13: 40$, covering the period of detection of re ected protons between 12:40 and 13:30 as recognized at $<\sim 0.5 \mathrm{keV}$ in the IMA spectrogram. The magnetic uctuations began earlier than the detection of the re ected ions. The start time of the magnetic uctuations corresponds to the time when the spacecraft got out from the wake and into the solar wind. This is because the waves generated by the re ected ions mainly on the dayside of the moon were convected down by the solar wind to the nightside to be detected by Kaguya. The magnetic uctuations also persisted after the disappearance of the re ected ions until the spacecraft entered into the wake. A similar pattern was observed for the next two revolutions.

Figure 7 shows that the uctuations were weak during the period from 13:15 to $13: 35$, despite the fact that the re ected protons were continuously observed. A part of the period (13:22-13:35) might be related with the absence of the magnetic connection to the lunar surface, but the period of the disconnection was much shorter. It is not surprising that the periods of absence of the magnetic connection and weak uctuations disagree because the propagation of the waves was not exactly parallel to the magnetic eld. The reason for the depression of the uctuation is not known, and any attempt to determine it would require a simulation study of the trajectories of the re ected particles and the mechanism of the excitation of the waves in which the con guration of the magnetic eld would play a crucial role.

Although the non-monochromatic whistlers near the moon and the whistler waves upstream of the Earth's bow shock resemble each other in terms of frequency range, they are quite different in bandwidth and polarization. The whistlers upstream from the bow shock were a circularly polarized, monochromatic wave, while the nonmonochromatic whistler waves near the moon show no peak frequency or preferred polarization. The wide range of frequency may be explained by the dispersion of the velocity of the re ected protons. According to Saito et al. (2008), the protons were scattered into various directions rather than re ected specularly. The velocity component parallel to the magnetic eld $\left(V_{i \|}-V_{\mathrm{sw} \|}\right)$ would be distributed over a signi cant range, as illustrated in Fig. 6(c). The resonant condition and thus the resonant frequency depend on $\left(V_{i \|}-V_{\mathrm{sw} \|}\right)$. Figure 8 shows the resonant conditions for Eq. (3) for upstream-propagating waves with reected ions, represented by two lines with different inclinations $\left|\left(V_{i \|}-V_{\mathrm{sw} \|}\right) \cos \theta_{k B}\right|$, together with a dispersion curve of the whistler wave

$$
\frac{c k}{\omega}=\frac{\omega_{p}}{\sqrt{\omega\left(\Omega_{e} \cos \theta_{k B}\right)}} .
$$

The lines cross the dispersion curve at different angular frequencies $\omega$, depending on the inclination. Thus, it is expected that the scattered protons might cause cyclotron resonance over a wide range of frequency.

Here we have assumed that whistler waves with a variety of $(\omega, \mathbf{k})$ would be generated that would correspond to a range of resonant velocities of the re ected protons and that they co-exist in the solar wind ow near the moon. However, it would require further theoretical or numerical work to determine whether the whistler mode would become unstable over the wide range of frequency as observed here.

The highest resonant frequency is given by the steepest line in Fig. 8. According to Saito et al. (2008), the energy of the re ected protons are $70 \%$ of the incident solar wind, that is, $\left|\mathbf{V}_{i}\right| \sim 0.84 V_{\mathrm{sw}}$. Therefore, we can estimate the largest inclination of the order of $1.84 V_{\mathrm{sw}} \cos \theta_{k B}$. A solar wind speed of $\sim 350 \mathrm{~km} \mathrm{~s}^{-1}$ and a plasma density of $\sim 6 \times 10^{6} \mathrm{~m}^{-3}$ were estimated from the upstream observation by ACE. The estimated electron density agreed with the plasma frequency obtained from the plasma wave measurement made by LRS/WFC instrument onboard Kaguya (courtesy of Y. Kasahara). By substituting these parameters together with $\Omega_{e}=2 \pi \times 1.4 \times 10^{2} \mathrm{~Hz}, \omega_{p}=$ $2 \pi \times 2.2 \times 10^{4} \mathrm{~Hz}$, and $\Omega_{i} / \Omega_{e}=0.54 \times 10^{-3}$ into Eqs. (3) and (6), we obtain the highest resonance frequency 

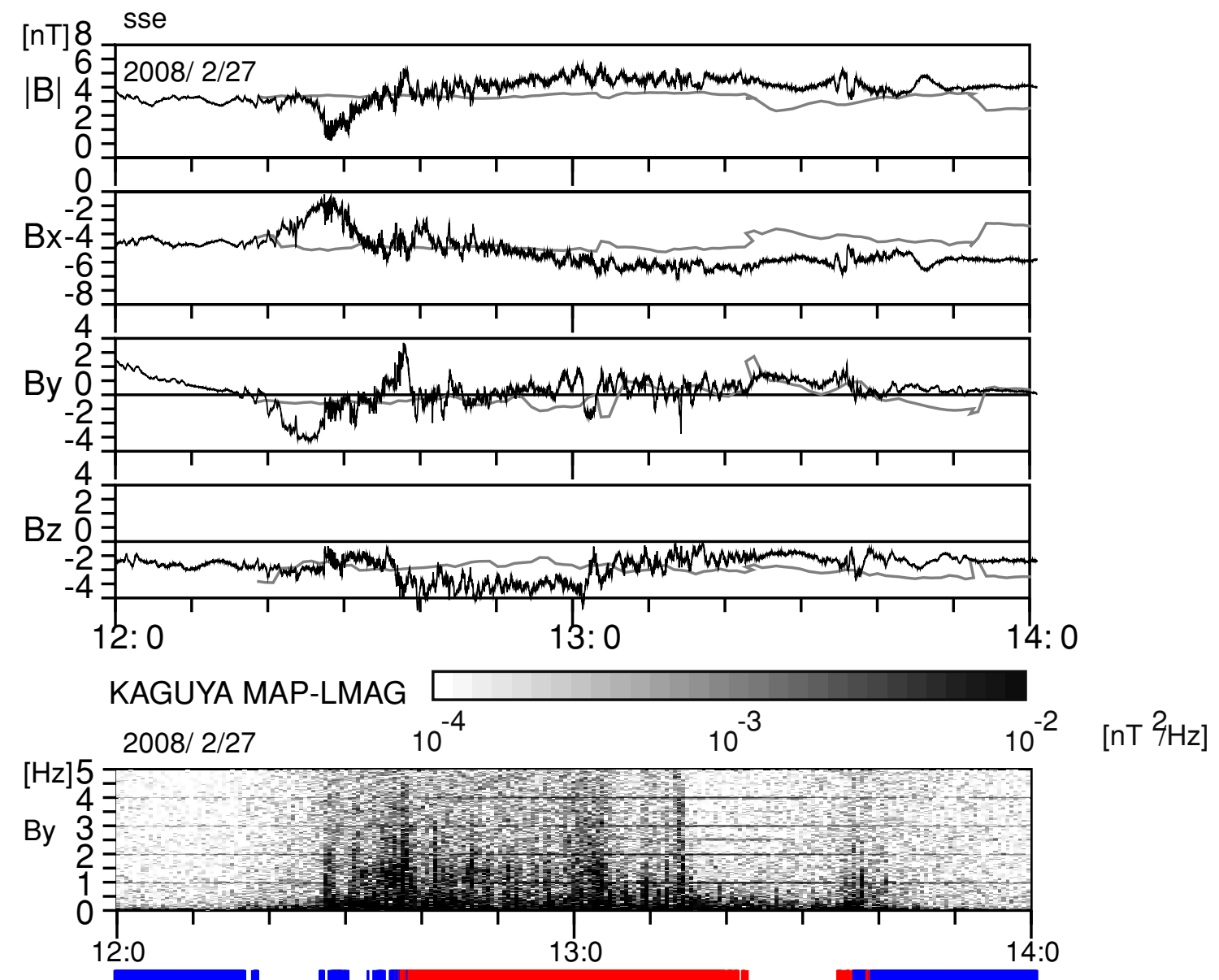

KAGUYA MAP-PACE $20080227120000-140000$

DIFFERENTIAL ENERGY FLUX
$\mathrm{cm}^{-2} \mathrm{~s}^{-1} \mathrm{str}^{-1} \mathrm{keV} / \mathrm{ke}$

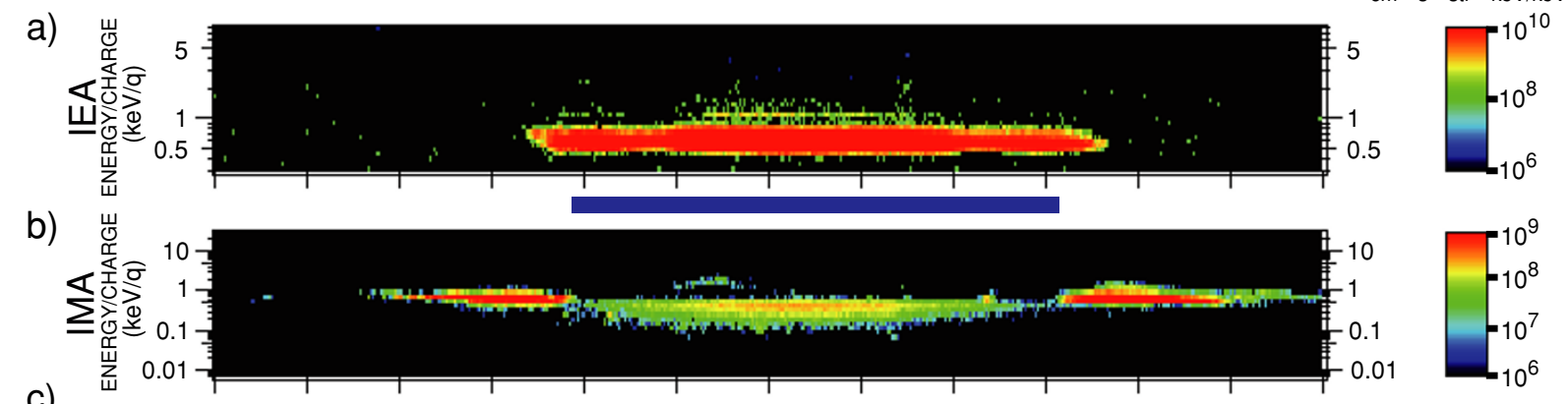

C)

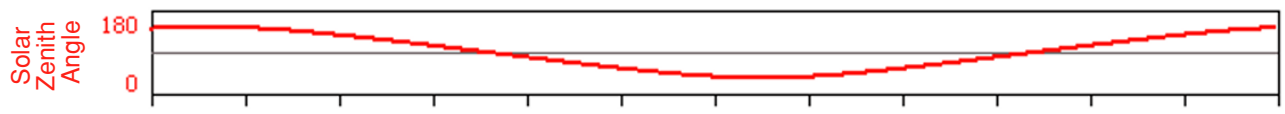

d)

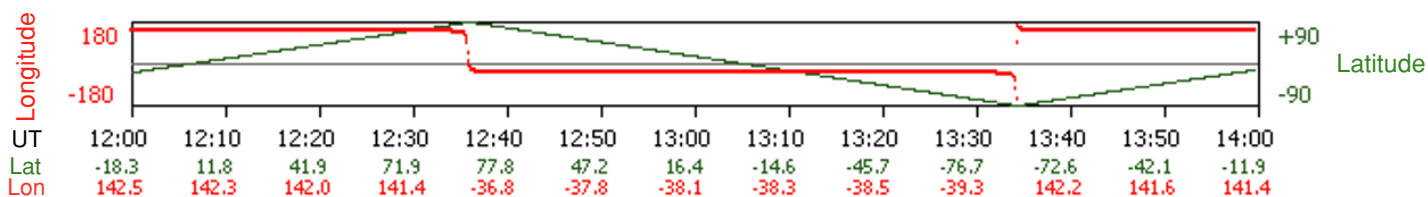

Fig. 7. The magnitude of the magnetic field $|\mathbf{B}|$ and the components $B_{x}, B_{y}, B_{z}$ observed by Kaguya (black) and ACE (gray, shifted to the Kaguya position using the solar wind speed), the dynamic spectrum for $B_{y}$ component, red (blue) bars which indicate the magnetic connection of the spacecraft to the dayside (nightside) surface of the moon, and the energy-time (E-t) spectrograms (adapted from figure 2 of Saito et al., 2008) obtained on February 27, 2008 by the MAP-PACE. (a) IEA instrument whose sensor was directed away from the moon, showing the direct observation of the solar wind at $\sim 0.6 \mathrm{keV}$ between 12:40 and 13:30 UT, and (b) IMA instrument which was looking into the dayside surface of the moon, showing the protons coming from the moon (marked with the blue bar above the spectrogram). Panels (c) and (d) show the solar zenith angle at Kaguya, and position of the spacecraft in ME coordinate system, respectively. 


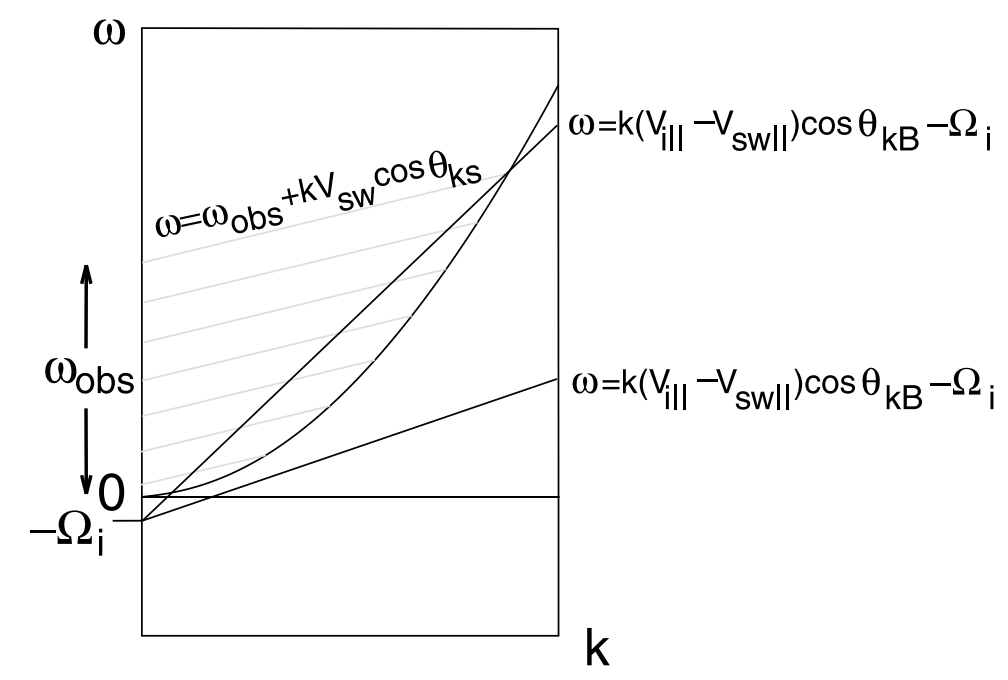

Fig. 8. Schematic illustrations of the dispersion relation of the whistler wave (the curve), the resonant condition (dark lines), and the equation of the Doppler shift (light lines). Note that $V_{i} \sim-0.84 V_{\mathrm{sw}}$.

$\omega / \Omega_{e}=0.1 \cos \theta_{k B}$ at $c k / \Omega_{e}=53$, which corresponds to $14 \cos \theta_{k B} \mathrm{~Hz}$. The waves would be Doppler shifted and then observed by Kaguya. Using Eq. (1) we obtain the highest frequency to be observed as $\left(14 \cos \theta_{k B}-8 \cos \theta_{k S}\right) \mathrm{Hz}$, where $\theta_{k S}$ is the angle between the wave number vector and the solar wind flow. This result seems to be consistent with the upper frequency of $\sim 10 \mathrm{~Hz}$ of the observed waves, as the wave number vector $\mathbf{k}$ was not parallel to the magnetic field nor the solar wind flow.

The whistler waves can propagate upstream against the solar wind if the group velocity $V_{\mathrm{g}}=2 c \sqrt{\omega \Omega_{e} \cos \theta_{k B}} / \omega_{p}$ overcomes the bulk flow as

$$
V_{\mathrm{g}} \cos \theta_{k S}>V_{\mathrm{sw}}
$$

This condition is satisfied at frequencies higher than 1.2/ $\cos \theta_{k B} \cos \theta_{k S} \mathrm{~Hz}$ for the parameters of February 27, 2008. In other words, low-frequency components of the non-monochromatic waves whose frequency is less than $1.2 \mathrm{~Hz}$ can not come from downstream of the spacecraft. They must be generated upstream of the spacecraft and then convected down. Indeed, the altitude of Kaguya, $100 \mathrm{~km}$ above the lunar surface, seems to be too low with respect to the Larmour radius of the reflected ions, which is as large as $1000 \mathrm{~km}$. The ions can not rotate even one cycle of gyration before they reach the altitude of the spacecraft. It may therefore be difficult for the waves to grow through the cyclotron resonance before reaching the spacecraft.

\subsection{Some non-resonant instabilities}

Another possibility is that the broadband emission was due to some non-resonant instabilities. Injection of the reflected protons into the solar wind plasma causes relative cross-field electron-ion streaming in which modified twostream instability may be present (McBride et al., 1972; Wu et al., 1984; Matsukiyo and Scholer, 2003). The advantages of the modified two-stream instability is that it grows within a short period that is comparable to the lowerhybrid frequency and much shorter than the gyro period and that the fluctuations have a compressional component. The broadband emission may then be due to a cascade process
(Matsukiyo and Scholer, 2003; Gary et al., 2008). It would require further work to determine the generation mechanism as well as the growth rate of the whistler waves and the direction of the wave number $\mathbf{k}$ to be chosen.

\section{Conclusion}

The non-monochromatic, $0.03-$ to $10-\mathrm{Hz}$ fluctuations of the magnetic field were detected by Kaguya at an altitude of $100 \mathrm{~km}$ above the lunar surface when the spacecraft was in the solar wind. The magnetic fluctuations were the whistler mode wave generated by the protons reflected by the lunar surface. The waves were not propagating parallel to the magnetic field, and the fluctuations had a compressional component. There was no peak frequency nor prefered polarization. The observed frequency range can be explained by cyclotron resonance of the whistler waves with the protons reflected by the lunar surface; however, it is not known why they were not propagating parallel to the magnetic field. Another possibility that the cross field velocity difference between the reflected ions and incident solar wind particles may cause some nonresonant instability, such as modified two-stream instability. Further theoretical or numerical work are required to reveal the exact generation mechanism.

Acknowledgments. The authors are grateful to S. Machida, Y. Kasahara, K. Tsubouchi, M. Nishino, and S. Matsukiyo for valuable comments and discussion. The authors are thankful to Y. Saito for the $E$ - $t$ diagram in Fig. 7 and Y. Kasahara for the electron density measured by LRS/WFC. This work was supported by JSPS Grant-in-Aid for Scientific Research project 21540461.

\section{References}

Bosqued, J. M., N. Lormant, H. Rème, C. d'Uston, R. P. Lin, K. A. Anderson, C. W. Carlson, R. E. Ergun, D. Larson, J. McFadden, M. P. McCarthy, G. K. Parks, T. R. Sanderson, and K.-P. Wenzel, Moonsolar wind interaction: First results from the WIND/3DP experiment, Geophys. Res. Lett., 23, 1259-1262, 1996.

Colburn, D. S., R. G. Currie, J. D. Mihalov, and C. P. Sonett, Diamagnetic solar-wind cavity discovered behind moon, Science, 158, 1040-1042, 1967.

Fairfield, D. H., Bow shock associated waves observed in the far upstream interplanetary medium, J. Geophys. Res., 74, 3541-3553, 1969. 
Fair eld, D. H., Whistler waves observed upstream from collisionless shocks, J. Geophys. Res., 79, 1368-1378, 1974.

Farrell, W. M., R. J. Fitzenreiter, C. J. Owen, J. B. Byrnes, R. P. Lepping, K. W. Ogilvie, and F. Neubauer, Upstream ULF waves and energetic electrons associated with the lunar wake: Detection of precursor activity, Geophys. Res. Lett., 23, 1271-1274, 1996.

Gary, P., S. Saito, and H. Li, Cascade of whistler turbulence: Particlein-cell simulations, Geophys. Res. Lett., 35, L02104, doi:10.1029/ 2007/GL032327, 2008.

Halekas, J. S., D. A. Brain, D. L. Mitchell, R. P. Lin, and L. Harrison, On the occurrence of magnetic enhancements caused by solar wind interaction with lunar crustal elds, Geophys. Res. Lett., 33, L08106, doi:10.1029/2006GL025938, 2006a.

Halekas, J. S., D. A. Brain, D. L. Mitchell, and R. P. Lin, Whistler waves observed near lunar crustal magnetic sources, Geophys. Res. Lett., 33, L22104, doi:10.1029/2006GL027684, 2006b.

Lyon, E. F., H. S. Bridge, and J. H. Binsak, Explorer 35 plasma measurements in the vicinity of the moon, J. Geophys. Res., 72, 6113, 1967.

Matsukiyo, S. and M. Scholer, Modi ed two-stream instability in the foot of high Mach number quasi-perpendicular shocks, J. Geophys. Res., 108, 1459, doi:10.1029/2003JA010080, 2003.

McBride, J. B., E. Orr, J. P. Boris, and J. H. Orens, Theory and simulation of turbulent heating by the modi ed two-stream instability, Phys. Fluids, 15, 2367-2383, 1972 .

Nakagawa, T., Y. Takahashi, and M. Iizima, GEOTAIL observation of upstream ULF waves associated with lunar wake, Earth Planets Space, 55, 569-580, 2003.

Ness, N. F., K. W. Behannon, H. E. Taylor, and Y. C. Whang, Perturbations of the interplanetary magnetic eld by the lunar wake, J. Geophys. Res., 73, 3421-3440, 1968.

Nishino, M. N., M. Fujimoto, K. Maezawa, Y. Saito, S. Yokota, K. Asamura, T. Tanaka, H. Tsunakawa, M. Matsushima, F. Takahashi, T. Terasawa, H. Shibuya, and H. Shimizu, Solar-wind proton access deep into the near-Moon wake, Geophys. Res. Lett., 36, L16103, doi:10.1029/2009GL039444, 2009.

Ogilvie, K. W., J. T. Steinberg, R. T. Fitzenreiter, C. J. Owen, A. J. Lazarus, W. M. Farrell, and R. B. Torbert, Observation of the lunar plasma wake from the WIND spacecraft on December 27, 1994, Geophys. Res. Lett.,
23, 1255-1258, 1996.

Owen, C. J., R. P. Lepping, K. W. Ogilvie, J. A. Slavin, W. M. Farrell, and J. B. Byrnes, The lunar wake at $6.8 \mathrm{R}_{L}$ : WIND magnetic eld observations, Geophys. Res. Lett., 23, 1263-1266, 1996.

Russell, C. T., Planetary upstream waves, in Solar Wind Sources of Magnetospheric Ultra-low-frequency Waves, edited by M. J. Engebretson, K. Takahashi and M. Scholer, AGU, Washington, D.C., 1994a.

Russell, C. T., Magnetospheric and solar wind studies with co-orbiting spacecraft, in Solar System Plasmas in Space and Time, edited by J. L. Burch and J. H. Waite, Jr., AGU, Washington, D.C., 1994b.

Saito, Y., S. Yokota, K. Asamura, T. Tanaka, R. Akiba, M. Fujimoto, H. Hasegawa, H. Hayakawa, M. Hirahara, M. Hoshino, S. Machida, T. Mukai, T. Nagai, T. Nagatsuma, M. Nakamura, K.-I. Oyama, E. Sagawa, S. Sasaki, K. Seki, and T. Terasawa, Low-energy charged particle measurement by MAP-PACE onboard SELENE, Earth Planets Space, 60, 375-385, 2008.

Schubert, G. and B. R. Lichtenstein, Observations of moon-plasma interactions by orbital and surface experiments, Rev. Geophys. Space Phys., 12, 592-626, 1974

Shimizu, H., F. Takahashi, N. Horii, A. Matsuoka, M. Matsushima, H. Shibuya, and H. Tsunakawa, Ground calibration of the highsensitivity SELENE lunar magnetometer LMAG, Earth Planets Space, 60, 353-363, 2008

Sonnerup, B. U. Ö. and L. J. Cahill, Jr., Magnetopause structure and attitude from Explorer 12 observations, J. Geophys. Res., 72, 171-183, 1967.

Takahashi, F., H. Shimizu, M. Matsushima, H. Shibuya, A. Matsuoka, S. Nakazawa, Y. Iijima, H. Otake, and H. Tsunakawa, In-orbit calibration of the lunar magnetometer onboard SELENE (KAGUYA), Earth Planets Space, 61, 1269-1274, 2009.

Wu, C. S., Y. M. Zhou, S. T. Tsai, S. C. Guo, D. Winske, and K. Papadopoulos, A kinetic cross- eld streaming instability, Phys. Fluids, 26, 1259-1267, 1984

T. Nakagawa (e-mail: nakagawa@tohtech.ac.jp), F. Takahashi, H. Tsunakawa, H. Shibuya, H. Shimizu, and M. Matsushima 